\title{
Restricted Flows and the Soliton Equation with Self-Consistent Sources ${ }^{\star}$
}

\author{
Runliang $\operatorname{LIN}^{\dagger}$, Haishen $Y A O^{\ddagger}$ and Yunbo ZENG ${ }^{\dagger}$ \\ $\dagger$ Department of Mathematical Sciences, Tsinghua University, Beijing 100084, P.R. China \\ E-mail: rlin@math.tsinghua.edu.cn,yzeng@math.tsinghua.edu.cn \\ URL: http://faculty.math.tsinghua.edu.cn/faculty/ rlin/ \\ $\ddagger$ Dept. of Math and Computer Science, QCC, The City University of New York, USA \\ E-mail: hyao@qcc.cuny.edu
}

Received October 28, 2006, in final form December 22, 2006; Published online December 30, 2006

Original article is available at http://www.emis.de/journals/SIGMA/2006/Paper096/

\begin{abstract}
The KdV equation is used as an example to illustrate the relation between the restricted flows and the soliton equation with self-consistent sources. Inspired by the results on the Bäcklund transformation for the restricted flows (by V.B. Kuznetsov et al.), we constructed two types of Darboux transformations for the KdV equation with self-consistent sources (KdVES). These Darboux transformations are used to get some explicit solutions of the KdVES, which include soliton, rational, positon, and negaton solutions.
\end{abstract}

Key words: the KdV equation with self-consistent sources; restricted flows; Lax pair; Darboux transformation; soliton solution

2000 Mathematics Subject Classification: 35Q51; 35Q53; 37K10

\section{Introduction}

The nonlinear evolution equation with sources has many applications in physics, such as hydrodynamics, plasma physics, solid state physics (see, e.g., [1, 2, 3, 4, 5, 6, 7, 8, 9, 10, 11]). Some $(1+1)$-dimensional systems of this kind can be written in bi-Hamiltonian form by changing the role of $x$ and $t[12,13]$. In recent years, certain equations with self-consistent sources have been studied by the inverse scattering method [1, 3, 5, 6], Darboux transformation [7, 8], and Hirota method $[9,10,11]$. Several types of solutions have been obtained.

In early works on soliton equations with self-consistent sources (SESCSs), since the Lax pairs of these systems were not obtained explicitly, the use of the inverse scattering method was complicated and needed special skills [1]. Because the restricted flows of soliton equations are just the stationary problems of SESCSs and the Lax pairs of restricted flows can always be deduced from the adjoint representation of soliton equations [14], a natural and simple way to deduce the auxiliary linear problems of SESCSs was discovered [4], and then a systematical approach to solve SESCSs by inverse scattering method was developed [5, 6].

The Hirota method has been used to construct several types of solutions for some SESCSs $[9,10]$. Recently, X.B. Hu and his colleagues developed a new procedure to construct some systems with self-consistent sources (see, e.g., [11]). Especially, some discrete systems with sources were studied there.

Darboux transformation is a powerful tool to construct some solutions of the differential equations [15]. Some SESCSs have been studied by Darboux transformation (see [7, 8] and the

\footnotetext{
*This paper is a contribution to the Vadim Kuznetsov Memorial Issue "Integrable Systems and Related Topics". The full collection is available at http://www.emis.de/journals/SIGMA/kuznetsov.html
} 
references therein). In [7], the KdV equation with self-consistent sources (KdVES) is studied by the generalized binary Darboux transformation, whose reduction to the form of Darboux transformation for the original KdV equation (without source) is not explicit. Some soliton, positon, and negaton solutions of the KdVES are obtained in [7]. W.X. Ma introduced complexiton solution to some soliton equations and showed that the complexiton solution of the KdVES can be obtained by Darboux transformation [8]. Notice the close relation between the restricted flows and the SESCSs, and stimulated by the study on the Bäcklund transformations for the restricted flows, which were studied on the basis of Darboux-Crum transformation by V.B. Kuznetsov et al. [16, 17, 18], two types of Darboux transformations for the KdVES are constructed in this paper. The first type of Darboux transformation is not a binary Darboux transformation, but it also enables us to obtain the soliton and rational solutions of the KdVES. The second type of Darboux transformation enables us to obtain the positon and negaton solutions of the KdVES. Some special cases of the solutions of the KdVES obtained in this paper reduce to some known solutions in other papers $[5,7]$.

The paper is organized as follows. In Section 2, the restricted flows of the KdV hierarchy and the KdV hierarchy with self-consistent sources are constructed. In Section 3, the Lax pair for the restricted flows and the auxiliary linear problems for the KdVES are deduced. In Section 4, two types of Darboux transformations for the KdVES are constructed, then some solutions of the KdVES are obtained. Finally, we summarize the main results of this paper in Section 5.

\section{The restricted flows and the KdVES}

Consider the Schrödinger equation

$$
\phi_{x x}+(\lambda+u) \phi=0,
$$

where $\phi$ and $u$ are functions of $x$ and $t, \lambda$ is a spectral parameter. Equation (2.1) can be written in the matrix form

$$
\left(\begin{array}{c}
\phi \\
\phi_{x}
\end{array}\right)_{x}=U\left(\begin{array}{c}
\phi \\
\phi_{x}
\end{array}\right), \quad U=\left(\begin{array}{cc}
0 & 1 \\
-\lambda-u & 0
\end{array}\right) .
$$

The adjoint representation of (2.2) reads [19]

$$
V_{x}=[U, V] \equiv U V-V U .
$$

Set

$$
V=\sum_{i=0}^{\infty}\left(\begin{array}{cc}
a_{i} & b_{i} \\
c_{i} & -a_{i}
\end{array}\right) \lambda^{-i} .
$$

Equation (2.3) yields

$$
\begin{aligned}
& a_{0}=b_{0}=0, \quad c_{0}=-4, \quad a_{1}=0, \quad b_{1}=4, \quad c_{1}=-2 u, \\
& a_{2}=u_{x}, \quad b_{2}=-2 u, \quad c_{2}=\frac{1}{2}\left(u_{x x}+u^{2}\right), \quad \ldots,
\end{aligned}
$$

and in general for $k=1,2, \ldots$,

$$
a_{k}=-\frac{1}{2} b_{k, x}, \quad b_{k+1}=L b_{k}=-\frac{1}{2} L^{k-1} u, \quad c_{k}=-\frac{1}{2} b_{k, x x}-b_{k+1}-b_{k} u,
$$

where

$$
L=-\frac{1}{4} D^{2}-u+\frac{1}{2} D^{-1} u_{x}, \quad D=\frac{\partial}{\partial x}, \quad D D^{-1}=D^{-1} D=1 .
$$


Set

$$
V^{(n)}=\sum_{i=0}^{n+1}\left(\begin{array}{cc}
a_{i} & b_{i} \\
c_{i} & -a_{i}
\end{array}\right) \lambda^{n+1-i}+\left(\begin{array}{cc}
0 & 0 \\
b_{n+2} & 0
\end{array}\right),
$$

and take

$$
\left(\begin{array}{c}
\phi \\
\phi_{x}
\end{array}\right)_{t_{n}}=V^{(n)}(u, \lambda)\left(\begin{array}{c}
\phi \\
\phi_{x}
\end{array}\right)
$$

Then the compatibility condition of equations (2.2) and (2.5) gives rise to the KdV hierarchy

$$
u_{t_{n}}=D \frac{\delta H_{n}}{\delta u} \equiv-2 b_{n+2, x}, \quad n=0,1, \ldots,
$$

where $H_{n}=\frac{4 b_{n+3}}{2 n+3}$. We have

$$
\frac{\delta \lambda}{\delta u}=\phi^{2}, \quad L \phi^{2}=\lambda \phi^{2} .
$$

The high-order restricted flows of the KdV hierarchy consist of the equations obtained from the spectral problem (2.1) for $N$ distinct $\lambda_{j}$ and the restriction of the variational derivatives for conserved quantities $H_{n}$ and $\lambda_{j}$ [4]

$$
\begin{aligned}
& \frac{\delta H_{n}}{\delta u}-2 \sum_{j=1}^{N} \frac{\delta \lambda_{j}}{\delta u} \equiv-2 b_{n+2}-2 \sum_{j=1}^{N} \phi_{j}^{2}=0, \\
& \phi_{j, x x}+\left(\lambda_{j}+u\right) \phi_{j}=0, \quad j=1, \ldots, N,
\end{aligned}
$$

where $n=0,1, \ldots$

The KdV hierarchy with self-consistent sources is given by[4]

$$
\begin{aligned}
& u_{t_{n}}=D\left[\frac{\delta H_{n}}{\delta u}-2 \sum_{j=1}^{N} \frac{\delta \lambda_{j}}{\delta u}\right] \equiv D\left[-2 b_{n+2}-2 \sum_{j=1}^{N} \phi_{j}^{2}\right], \\
& \phi_{j, x x}+\left(\lambda_{j}+u\right) \phi_{j}=0, \quad j=1, \ldots, N,
\end{aligned}
$$

where $\lambda_{j}$ are distinct.

In this paper, we concentrate on the case $n=1$ and denote $t \equiv t_{1}$, the equation (2.8) gives the KdV equation with self-consistent sources (KdVES)

$$
\begin{aligned}
& u_{t}=-\left(6 u u_{x}+u_{x x x}\right)-2 \frac{\partial}{\partial x} \sum_{j=1}^{N} \phi_{j}^{2}, \\
& \phi_{j, x x}+\left(\lambda_{j}+u\right) \phi_{j}=0, \quad j=1, \ldots, N .
\end{aligned}
$$

\section{The Lax pair for the restricted flows and that for the KdVES}

Set $\Phi=\left(\phi_{1}, \ldots, \phi_{N}\right)^{T}$. According to equations (2.4), (2.6) and (2.7), we denote

$$
\begin{aligned}
& \widetilde{a}_{i}=a_{i}, \quad \widetilde{b}_{i}=b_{i}, \quad \widetilde{c}_{i}=c_{i}, \quad i=0,1, \ldots, n+1, \\
& \widetilde{b}_{n+2+i}=-\left\langle\Lambda^{i} \Phi, \Phi\right\rangle, \quad i=0,1,2, \ldots, \\
& \widetilde{a}_{n+2+i}=-\frac{1}{2} \widetilde{b}_{n+2+i, x}=\left\langle\Lambda^{i} \Phi, \Phi_{x}\right\rangle,
\end{aligned}
$$




$$
\widetilde{c}_{n+2+i}=-\frac{1}{2} \widetilde{b}_{n+2+i, x x}-\widetilde{b}_{n+3+i}-\widetilde{b}_{n+2+i} u=\left\langle\Lambda^{i} \Phi_{x}, \Phi_{x}\right\rangle .
$$

Then

$$
\begin{aligned}
N^{(n)} & \equiv\left(\begin{array}{ll}
A^{(n)} & B^{(n)} \\
C^{(n)} & D^{(n)}
\end{array}\right)=\lambda^{n+1} \sum_{k=0}^{\infty}\left(\begin{array}{cc}
\widetilde{a}_{k} & \widetilde{b}_{k} \\
\widetilde{c}_{k} & -\widetilde{a}_{k}
\end{array}\right) \lambda^{-k} \\
& =\sum_{k=0}^{n+1}\left(\begin{array}{cc}
a_{k} & b_{k} \\
c_{k} & -a_{k}
\end{array}\right) \lambda^{n+1-k}+\sum_{j=1}^{N} \frac{1}{\lambda-\lambda_{j}}\left(\begin{array}{cc}
\phi_{j} \phi_{j, x} & -\phi_{j}^{2} \\
\phi_{j, x}^{2} & -\phi_{j} \phi_{j, x}
\end{array}\right),
\end{aligned}
$$

also satisfies the adjoint representation (2.3), i.e.

$$
N_{x}^{(n)}=\left[U, N^{(n)}\right] .
$$

In fact equation (3.1) gives rise to the Lax representation of the restricted flow (2.7).

Since the high-order restricted flows (2.7) are just the stationary equations of the KdV hierarchy with self-consistent sources (2.8), it is obvious that the zero-curvature representation for the KdV hierarchy with self-consistent sources (2.8) is given by

$$
U_{t_{n}}-N_{x}^{(n)}+\left[U, N^{(n)}\right]=0,
$$

with the auxiliary linear problems

$$
\left(\begin{array}{c}
\psi \\
\psi_{x}
\end{array}\right)_{x}=U\left(\begin{array}{c}
\psi \\
\psi_{x}
\end{array}\right), \quad\left(\begin{array}{c}
\psi \\
\psi_{x}
\end{array}\right)_{t_{n}}=N^{(n)}\left(\begin{array}{c}
\psi \\
\psi_{x}
\end{array}\right),
$$

or equivalently

$$
\begin{aligned}
& \psi_{x x}+(\lambda+u) \psi=0, \\
& \psi_{t_{n}}=A^{(n)} \psi+B^{(n)} \psi_{x}=\sum_{l=0}^{n+1}\left(a_{l} \psi+b_{l} \psi_{x}\right) \lambda^{n+1-l}+\sum_{j=1}^{N} \frac{1}{\lambda-\lambda_{j}} \phi_{j}\left(\phi_{j, x} \psi-\phi_{j} \psi_{x}\right) .
\end{aligned}
$$

In particular, the system (3.2) for $n=1$ gives the auxiliary linear problem for the KdVES (2.9)

$$
\begin{aligned}
& \psi_{x x}+(\lambda+u) \psi=0, \\
& \psi_{t}=u_{x} \psi+(4 \lambda-2 u) \psi_{x}+\sum_{j=1}^{N} \frac{1}{\lambda-\lambda_{j}} \phi_{j}\left(\phi_{j, x} \psi-\phi_{j} \psi_{x}\right) .
\end{aligned}
$$

The compatibility condition of (3.3a) and (3.3b) gives the KdVES (2.9) under the assumption $(2.9 \mathrm{~b})$.

\section{The Darboux transformation for the KdVES}

The Bäcklund transformation for the restricted flows of KdV hierarchy (2.7) has been studied by Kuznetsov et al. [16, 17, 18]. These transformations can be extended to construct the Darboux transformation for the KdVES. Two types of Darboux transformations and some solutions for the KdVES will be constructed in this section.

In the following, we use $W\left(g_{1}, \ldots, g_{m}\right)$ to denote the Wronskian determinant for functions $g_{1}(x), g_{1}(x), \ldots, g_{m}(x)$, i.e.,

$$
W\left(g_{1}, g_{2}, \ldots, g_{m}\right)=\left|\begin{array}{cccc}
g_{1} & g_{2} & \cdots & g_{m} \\
\partial_{x} g_{1} & \partial_{x} g_{2} & \cdots & \partial_{x} g_{m} \\
\cdots & \cdots & \cdots & \cdots \\
\partial_{x}^{m-1} g_{1} & \partial_{x}^{m-1} g_{2} & \cdots & \partial_{x}^{m-1} g_{m}
\end{array}\right| .
$$




\subsection{First type of Darboux transformation for the KdVES}

Proposition 1. Suppose $u, \phi_{1}, \ldots, \phi_{N}$, is a solution of the $\operatorname{KdVES}(2.9), \psi, u, \phi_{1}, \ldots, \phi_{N}$, satisfy the linear problem (3.3). If the functions $f\left(x, t, \lambda_{N+1}\right)$ and $g\left(x, t, \lambda_{N+1}\right)$ are two solutions of (3.3) with $\lambda=\lambda_{N+1}$ (where $\lambda_{N+1} \neq \lambda_{j}$ for $\left.j=1, \ldots, N\right)$, and $W(f, g) \neq 0$, then the following functions satisfy the linear problem (3.3) (with $N$ replaced by $N+1$ )

$$
\begin{aligned}
& \widetilde{\psi}=\frac{W(S, \psi)}{S}, \quad \widetilde{u}=u+2 \partial_{x}^{2} \ln S, \\
& \widetilde{\phi}_{j}=\frac{1}{\sqrt{\lambda_{j}-\lambda_{N+1}}} \frac{W\left(S, \phi_{j}\right)}{S}, \quad j=1, \ldots, N, \quad \widetilde{\phi}_{N+1}=\sqrt{\frac{C_{t}}{W(f, g)}} \frac{W(S, f)}{S},
\end{aligned}
$$

where $S=C(t) f\left(x, t, \lambda_{N+1}\right)+g\left(x, t, \lambda_{N+1}\right), C(t)$ is a differentiable function of $t$, and $C_{t}=\frac{d C}{d t}$.

Proof. It can be proved by direct computation.

It is easy to check that $\widetilde{\phi}_{j}(j=1, \ldots, N+1)$ in (4.1) satisfies (2.9b) under the assumption in Proposition 1. That means, we can use the Darboux transformation (4.1) (with $C(t)$ being variant in $t$ ) to obtain a solution of the KdVES (2.9) with $N$ replaced by $N+1$. If we fix the function $C(t)$ in (4.1) to be a constant, then the Darboux transformation (4.1) for the case $N=0$ reduces to the Darboux transformation for the original KdV equation (without source).

\subsubsection{Soliton solution}

It is easy to see that the KdVES (2.9) with $N=1$ and $\lambda_{1}=0$ has the following solution

$$
u=0, \quad \phi_{1}=\eta(t) .
$$

With the above $u$ and $\phi_{1}$, we take two solutions of (3.3) for $\lambda=-k^{2}$ (where $k>0$ ) as

$$
f=\exp (k x-a(t)), \quad g=\exp (-k x+a(t))
$$

where $a(t)$ is a differentiable function of $t$ and

$$
\frac{d a}{d t}=4 k^{3}-\frac{\eta(t)^{2}}{k} .
$$

Then use the Darboux transformation (4.1) with $C(t)=\exp (-2 z(t))$, where $z(t)$ is a differentiable function of $t$, we get a solution of the KdVES (2.9) with $N=2$

$$
\begin{aligned}
& \widetilde{u}=2 k^{2} \operatorname{sech}^{2}(k x-a(t)-z(t)), \quad \widetilde{\phi}_{1}=-\eta(t) \tanh (k x-a(t)-z(t)), \\
& \widetilde{\phi}_{2}=\sqrt{k \frac{d z}{d t}} \operatorname{sech}(k x-a(t)-z(t)),
\end{aligned}
$$

where $a(t)$ is given by (4.2). The velocity of propagation of this soliton solution can be modified by the choice of the function $z(t)[1,5]$. This phenomena is different with the case of the original $\mathrm{KdV}$ equation where the velocity is proportional to the amplitude of soliton. One should notice that $\left|\widetilde{\phi}_{1}\right| \rightarrow|\eta(t)|$ when $|x| \rightarrow \pm \infty$, this kind of source is studied less in the references. In addition, if we set $\eta(t) \equiv 0$, the solution (4.3) reduces to the soliton solution obtained in some other papers $[1,5]$. 


\subsubsection{Rational solution}

The KdVES (2.9) with $N=0$ has a trivial solution $u=0$. Take two solutions of (3.3) with $u=0$ and $\lambda=0$ as follows

$$
f=1, \quad g=x,
$$

then use the Darboux transformation (4.1), we get a solution of the KdVES (2.9) with $N=1$

$$
\widetilde{u}=\frac{-2}{(x+C(t))^{2}}, \quad \widetilde{\phi}_{1}=\frac{-\sqrt{C_{t}}}{(x+C(t))} .
$$

It is a rational solution, and the poles of the solution are variant with respect to the choice of function $C(t)$.

\subsection{Second type of Darboux transformation for the KdVES}

In the following, we will construct another type of Darboux transformation for the KdVES (2.9), which enables us to obtain positon and negaton solutions of the KdVES (2.9).

Proposition 2. Suppose $u, \phi_{1}, \ldots, \phi_{N}$, is a solution of the $K d V E S(2.9), \psi, u, \phi_{1}, \ldots, \phi_{N}$, satisfy the linear problem (3.3). If the functions $f\left(x, t, \lambda_{N+1}\right)$ and $g\left(x, t, \lambda_{N+1}\right)$ are two solutions of (3.3) with $\lambda=\lambda_{N+1}$ (where $\lambda_{N+1} \neq \lambda_{j}$ for $\left.j=1, \ldots, N\right)$, and $W(f, g) \neq 0$ ), then the following functions satisfy the linear problem (3.3) (with $N$ replaced by $N+1$ )

$$
\begin{aligned}
& \widetilde{\Psi}=\frac{W(g, T, \Psi)}{W(g, T)}, \quad \widetilde{u}=u+2 \partial_{x}^{2} \ln W(g, T), \\
& \widetilde{\phi}_{j}=\frac{1}{\lambda_{j}-\lambda_{N+1}} \frac{W\left(g, T, \phi_{j}\right)}{W(g, T)}, \quad j=1, \ldots, N, \quad \widetilde{\phi}_{N+1}=\sqrt{\frac{C_{t}}{W(f, g)}} \frac{W(g, T, f)}{W(g, T)},
\end{aligned}
$$

where $T=C(t) f\left(x, t, \lambda_{N+1}\right)+\partial_{N+1} g\left(x, t, \lambda_{N+1}\right)$ and $C(t)$ is a differentiable function of $t$.

Proof. It can be proved by direct computation.

It is easy to check that $\widetilde{\phi}_{j}(j=1, \ldots, N+1)$ in (4.4) satisfies $(2.9 \mathrm{~b})$ under the assumption in Proposition 2. That means, we can use the Darboux transformation (4.1) (with $C(t)$ being variant in $t$ ) to obtain a solution of the KdVES (2.9) with $N$ replaced by $N+1$.

\subsubsection{Positon solution}

The KdVES (2.9) with $N=1$ and $\lambda_{1}=0$ has a trivial solution

$$
u=0, \quad \phi_{1}=\sqrt{\frac{d \eta(t)}{d t}},
$$

where $\eta(t)$ is a differentiable function of $t$. With the above $u$ and $\phi_{1}$, we take two solutions of (3.3) for $\lambda=k^{2}$ (where $k>0$ ) as

$$
f=\cos \Theta, \quad g=\sin \Theta, \quad \Theta=k x+4 k^{3} t-\frac{\eta(t)}{k}+b(k),
$$

where $b(k)$ is a differentiable function of $k$. By using the Darboux transformation (4.4), we get a solution of KdVES (2.9) with $N=2$

$$
\widetilde{u}=\frac{32 k^{2}\left(2 k^{2} \gamma \cos \Theta-\sin \Theta\right) \sin \Theta}{\left(4 k^{2} \gamma-\sin (2 \Theta)\right)^{2}},
$$




$$
\widetilde{\phi}_{1}=\frac{-\sqrt{\eta_{t}}\left(4 k^{2} \gamma+\sin (2 \Theta)\right)}{4 k^{2} \gamma-\sin (2 \Theta)}, \quad \widetilde{\phi}_{2}=\frac{4 k \sqrt{k C_{t}} \sin \Theta}{4 k^{2} \gamma-\sin (2 \Theta)}
$$

where

$$
\gamma=C(t)+\frac{1}{2 k} \partial_{k} \Theta
$$

This is a positon solution (see [7] and the references therein). If we set $\phi_{1}=0$, then the solution (4.5) reduces to the one given in [7].

\subsubsection{Negaton solution}

The KdVES (2.9) with $N=1$ and $\lambda_{1}=0$ has a trivial solution

$$
u=0, \quad \phi_{1}=\sqrt{\frac{d \eta(t)}{d t}},
$$

where $\eta(t)$ is a differentiable function of $t$. With the above $u$ and $\phi_{1}$, we take two solutions of (3.3) for $\lambda=-k^{2}$ (where $k>0$ ) as

$$
f=\cosh \Theta, \quad g=\sinh \Theta, \quad \Theta=k x-4 k^{3} t+\frac{\eta(t)}{k}+b(k),
$$

where $b(k)$ is a differentiable function of $k$. By using the Darboux transformation (4.4), we get a solution of KdVES (2.9) with $N=2$

$$
\begin{aligned}
& \widetilde{u}=\frac{8 k^{2}\left(2 k^{2} \gamma \cosh \Theta+\sinh \Theta\right) \sinh \Theta}{\left(2 k^{2} \gamma+\sinh \Theta \cosh \Theta\right)^{2}}, \\
& \widetilde{\phi}_{1}=\frac{\sqrt{\eta_{t}}\left(-2 k^{2} \gamma+\sinh \Theta \cosh \Theta\right)}{2 k^{2} \gamma+\sinh \Theta \cosh \Theta}, \quad \widetilde{\phi}_{2}=\frac{2 k \sqrt{k C_{t}} \sinh \Theta}{2 k^{2} \gamma+\sinh \Theta \cosh \Theta},
\end{aligned}
$$

where

$$
\gamma=C(t)-\frac{1}{2 k} \partial_{k} \Theta
$$

This is a negaton solution (see [7] and the references therein). If we set $\phi_{1}=0$, then the solution (4.6) reduces to the one given in [7] (notice that there is a typo in the expression of negaton solution $(4.2 \mathrm{~b})$ in [7]).

\section{Conclusion}

The $\mathrm{KdV}$ equation is used as an example to illustrate the relation between the restricted flows and the soliton equation with self-consistent sources (SESCSs). Since the restricted flows is just the stationary problem of the SESCSs and the Lax pair of the restricted flows can always be deduced from the adjoint representation of soliton equations, the auxiliary linear problem for the SESCSs can be easily obtained. Stimulated by the study on the Bäcklund transformation for the restricted flows (by Kuznetsov et al.) [16, 17, 18], two types of Darboux transformations for the KdVES are constructed in this paper. The first type of Darboux transformation is not a binary one, whose reduction relation to the form of Darboux transformation for the original $\mathrm{KdV}$ equation is shown. By the two types of Darboux transformation, some explicit solutions for the KdVES are obtained, which include soliton, rational, positon, and negation solutions. It is possible to construct complexiton solution of the KdVES by the Darboux transformation in this paper, we will study it in detail in the future work. 
This paper is submitted to the memorial volume for Vadim B. Kuznetsov. Dr. Kuznetsov was an expert in integrable systems. One of the authors (R.L. Lin) visited him at Leeds in 2002 at his invitation. R.L. Lin was deeply impressed that he was so kindly to help the younger researchers. As we know, he kept close contact with some Chinese researchers and he visited another author (Y.B. Zeng) at Tsinghua University in 2002. Also, he applied some financial aid to help several Chinese researchers to work in Leeds. Vadim left, but his smile is left in our memory.

\section{Acknowledgements}

The authors are grateful to the referees for the valuable comments. This work is supported by the Chinese Basic Research Project "Nonlinear Science". R.L. Lin is supported in part by "Scientific Foundation for Returned Overseas Chinese Scholars, Ministry of Education".

[1] Mel'nikov V.K., Integration method of the Korteweg-de Vries equation with a self-consistent source, Phys. Lett. A, 1988, V.133, 493-496.

[2] Shchesnovich V.S., Doktorov E.V., Modified Manakov system with self-consistent source, Phys. Lett. A, 1996, V.213, 23-31.

[3] Urazboev G.U., Khasanov A.B., Integrating the Korteweg-de Vries equation with a self-consistent source and "steplike" initial data, Theoret. and Math. Phys., 2001, V.129, 1341-1356.

[4] Zeng Y.B., New factorization of the Kaup-Newell hierarchy, Phys. D, 1994, V.73, 171-188.

[5] Lin R.L., Zeng Y.B., Ma W.X., Solving the KdV hierarchy with self-consistent sources by inverse scattering method, Phys. A, 2001, V.291, 287-298.

[6] Zeng Y.B., Ma W.-X., Lin R.L., Integration of the soliton hierarchy with self-consistent sources, J. Math. Phys., 2000, V.41, 5453-5489.

[7] Zeng Y.B., Shao Y.J., Xue W.M., Negaton and positon solutions of the soliton equation with self-consistent sources, J. Phys. A: Math. Gen., 2003, V.36, 5035-5043.

[8] Ma W.X., Complexiton solutions of the Korteweg-de Vries equation with self-consistent sources, Chaos Solitons Fractals, 2005, V.26, 1453-1458.

[9] Hu X.B., The higher-order KdV equation with a source and nonlinear superposition formula, Chaos Solitons Fractals, 1996, V.7, 211-215.

[10] Zhang D.J., Chen D.Y., The $N$-soliton solutions of the sine-Gordon equation with self-consistent sources, Phys. A, 2003, V.321, 467-481.

[11] Gegenhasi, Hu X.B., On a integrable differential-difference equation with a source, J. Nonlinear Math. Phys., 2006, V.13, 183-192.

[12] Zeng Y.B., Bi-Hamiltonian structure of JM hierarchy with self-consistent sources, Phys. A, 1999, V.262, 405-419.

[13] Zeng Y.B., Lin R.L., Wang X.H., Bi-Hamiltonian structure of the KdV hierarchy with self-consistent sources, Adv. Math. (China), 1998, V.27, 451-463.

[14] Zeng Y.B., Li Y.S., The deduction of the Lax representation for constrained flows from the adjoint representation, J. Phys. A: Math. Gen., 1993, V.26, L273-L278.

[15] Matveev V.B., Salle M.A., Darboux transformations and solitons, Berlin, Springer, 1991.

[16] Kuznetsov V.B., Sklyanin E.K., On Bäcklund transformations for many-body systems, J. Phys. A: Math. Gen., 1998, V.31, 2241-2251, solv-int/9711010.

[17] Hone A.N.W., Kuznetsov V.B., Ragnisco O., Bäcklund transformations for many-body systems related to KdV, J. Phys. A: Math. Gen., 1999, V.32, L299-L306, solv-int/9904003.

[18] Hone A.N.W., Kuznetsov V.B., Ragnisco O., Bäcklund transformations for the Hénon-Heiles and Garnier systems, in Proceedings of the SIDE III (1998, Sabaudia), Editors D. Levi and O. Ragnisco, CRM Proceedings and Lecture Notes Series, Vol. 25, American Mathematical Society, 2000, 231-235.

[19] Newell A.C., Solitons in mathematics and physics, Philadelphia, SIAM, 1985. 